\title{
Intersecciones entre las políticas sociales y las acciones de seguridad: elementos para analizar los cambios en la protección social en el contexto de crisis del capital
}

\section{Intersections between social policies and security actions: elements to analyze changes in social protection in the context of the capital crisis}

\author{
Alejandra Pastorini ${ }^{a}$
}

\begin{abstract}
Resumen
Este trabajo, que consisteen una reflexión teórica, tiene como objetivo problematizar algunos de los elementos entendidos como centrales para analizar los cambios producidos en las políticas sociales en las últimas décadas. Partimos de la premisa que el conjunto de transformaciones que se procesan en las políticas de protección social en los países capitalistas, a partir de los años 1980-90, es inseparable de la crisis del capital que detona en la década de 1970. A partir de ese momento el capital exige cambios en la organización de la máquina pública y en los mecanismos correctivos y de regulación del Estado. Estas redefiniciones contrarían el discurso de la minimización del Estado ya que implican en el crecimiento de la importancia que este complejo social tiene en el auxilio a los grandes negocios. Entender esos cambios es fundamental para pensar la centralidad que viene asumiendo los programas de transferencias monetarias focalizados en la pobreza y las acciones en el área denominada como seguridad apoyadas en el discurso del miedo y en la intensificación de la coerción.
\end{abstract}

Palabras clave: crisis del capital, estado, política social, protección social, seguridad.

\footnotetext{
Abstract

This work, which consists of a theoretical reflection, has as its objective to problematize some of the elements understood as central to analyze the changes produced in the social policies in the last decades. We start from the premise that the set of transformations that are taking place in the policies of social protection in the capitalist countries, from the years 1980-90, are inseparable from the crisis of the capital that detonated in the 1970s. At this moment capital demands changes in the organization of the public machine
}

a Universidade Federal do Rio de Janeiro (ESS-UFRJ), Brasil.

Correspondencia a:

alejandrapastorini@gmail.com

Recibido:

3o agosto 2018

Aceptado:

24 octubre 2018

Artículo publicado en acceso abierto bajo la Licencia Creative Commons.

\section{(c) (i)}

Cita:

Pastorini, A. (2018). Intersecciones entre las políticas sociales y las acciones de seguridad: elementos para analizar los cambios en la protección social en el contexto de crisis del capital. Kera Yvoty: reflexiones sobre la cuestión social, 3, 64-74. 
and in the corrective and regulatory mechanisms of the state. These redefinitions contradict the discourse of state minimization and that they imply in the growth of the importance that this social complex has in helping big business. Understanding these changes is fundamental to thinking about the centrality that has come to bear on the money transfer programs focused on poverty and actions in the area called security backed by the discourse of the medium and the intensification of coercion.

Keywords: capital crisis, state, social policy, social protection, security.

\section{Introducción}

Enlasúltimasdécadaslosmecanismos de protección social en el mundo capitalista pasan por importantes transformaciones. Más allá de las características particulares que esas estructuras de protección hayan tenido en cada país hasta los años 198090, es posible identificar en las últimas tres décadas algunos cambios que se orientan en una misma dirección y que se expresan en las alteraciones de las formas y particularidades que asumen las políticas sociales. Al mismo tiempo, esas transformaciones se manifiestan en las características que asumen los grupos y sectores destinatarios reales de las políticas públicas.

Desde los últimos años del siglo $\mathrm{xx}$ las acciones asistenciales (y dentro de estas los programas de transferencia monetaria) asumen un enorme protagonismo en términos políticos e ideológicos dentro del conjunto de las estrategias y políticas de protección social implementadas por los Estados nacionales. Estos programas en el área de asistencia que se caracterizan por ser territorializados y focalizados en los sectores más pauperizados se articulan, en los distintos espacios geográficos donde los usuarios viven, trabajan o realizan diversas actividades recreativas (principalmente en las periferias urbanas y suburbios), con los programas y acciones constitutivos de las denominadas políticas de seguridad pública.

Estas dos estrategias (denominadas aquí de asistencial y de seguridad) mismo que con características diferentes, poseen un fuerte carácter punitivo, controlador, moralizador y coercitivo de los sectores más empobrecidos $y$, en su conjunto, se transforman en un importante mecanismo de los Estados para administrar a la superpoblación relativa, o sea, para gestionar una creciente masa de trabajadores sobrantes desde el punto de vista de la acumulación del capital.

Estos cambios en las características de las políticas sociales se vinculan, por un lado, con la reducción del protagonismo del Estado en el área social. Principalmente, desde la segunda mitad de los años 1980, en el marco de los programas de las reformas neoliberales promovidas por los organismos multilaterales, ${ }^{1}$ el Estado fue disminuyendo su papel de proveedor directo de los mecanismos de protección en las áreas de educación, salud y previsión social (principales pilares de las estructuras de protectivas en el mundo capitalista hasta ese momento). Por otro lado, presenciamos el aumento de la participación del capital en el sector de servicios, alimentando los procesos de mercantilización de lo social; ese proceso retira el carácter de derecho y transforma a la educación, salud y a los beneficios de la previsión social (como jubilaciones y pensiones) en mercancías a ser compradas en el mercado.

La transformación de la protección social se expresa en el proceso de redefinición de los grupos y sectores protegidos y desprotegidos por el Estado. Principalmente en las últimas décadas podemos ver un cambio significativo en el

\footnotetext{
1 Para discutir la incidencia de los organismos multilaterales como Banco Mundial y Banco Interamericano de Desarrollo en las reforman neoliberales en América Latina puede ser consultado Pastorini y Galizia (2006).
} 
Pastorini, A. Intersecciones entre las políticas sociales y las acciones de seguridad: elementos para analizar los cambios en la protección social en el contexto de crisis del capital.

perfil de las personas que son protegidas por las políticas sociales; esa alteración en muchos casos se articula al aumento del desempleo y a la intensificación de la precarización del trabajo. También esos cambios en las características de los grupos que encuentran dificultades para poder tener acceso al conjunto de bienes, servicios y transferencias monetarias que dan forma concreta a las políticas sociales, en muchos casos se relaciona con los criterios de elegibilidad y condicionalidades impuestos por los programas sociales que acaban funcionando como mecanismos limitadores del acceso a las políticas sociales.

Ese proceso de reorganización de las estructuras de protección social se coloca como una tendencia mundial en las sociedades capitalistas. Pero es importante mencionar dos elementos. Primero, esa tendencia general se va a expresar con particularidades en las diferentes formaciones sociales y en las diversas coyunturas sociohistóricas. Segundo, la reorganización de los mecanismos de protección social asume un impacto devastador principalmente en los países que nunca convivieron con experiencia de Estados de bien estar amplias y consolidadas, como es el caso de la mayor parte de los países dependientes localizados en la periferia del capitalismo.

Mismo que en algunos países del cono sur de América Latina, como por ejemplo Brasil, Argentina, Chile y Uruguay ${ }^{2}$ se hayan desarrollado desde las primeras décadas del siglo XX diversas políticas sociales y consolidaron importantes leyes y derechos laborales, en esos países nunca se crearon sistemas de protección social inclusivos, amplios y con pretensiones universalistas; de esta forma se trata de estructuras limitadas en términos de redistribución y de las posibilidades de

2 Fleury (1994) realiza una importante síntesis de su investigación sobre la trayectoria de la protección social en diversos países de América Latina que le permite clasificar a estos países como pioneros debido a su temprana estructuración de los mecanismos de protección social. ampliación de la cobertura para el conjunto de las clases subalternas. ${ }^{3}$

A pesar de la selectividad y segmentación de la protección social, una parte de los trabajadores fabriles urbanos (en su mayoría con vínculos formales de empleo) y grandes sectores de la "clase media" de algunos de los países de esta región latinoamericana, por un largo periodo de tiempo y en algunas coyunturas específicas, participaron de las ventajas posibilitadas por la extracción del excedente económico en otros países de la región o en sectores específicos de la economía, sin que eso implicara necesariamente en una mejoría de la calidad de vida de la clase trabajadora en su conjunto en esas formaciones sociales. Esas ventajas fueron posibilitadas por el proceso de expansión del capital (principalmente en los contextos post guerras) y por la forma de inserción de estas naciones en la dinámica de la economía capitalista mundial, naciones que se tornaron atractivas para los países de economía avanzada por motivos variados (localización geográfica, riqueza natural, facilidades para explotar la fuerza de trabajo etc.). Esas particularidades y coyunturas sociales van a posibilitar la creación y ampliación de las políticas sociales y el reconocimiento jurídico de algunos derechos laborales y sociales.

Esta realidad revela el carácter desigual y combinado del desarrollo capitalista que se expresará también en el Estado (yen la forma jurídica a ésteasociada) y en los diversos mecanismos de regulación colocados en funcionamiento para crear las condiciones necesarias para la expansión del capital. O sea, la dinámica global del capital es inseparable de la consolidación de las desigualdades entre países, regiones, clases, grupos e individuos contrariando de esta forma la ilusión liberal del "bienestar de todos los ciudadanos" y del mito de la "igualdad y libertad de los individuos" justificadoras de la desigualdad estructural.

3 Esta discusión puede ser encontrada en Pastorini y Galizia (2012). 
Como indica Mészáros (2002), el Estado es la "única estructura correctiva compatible con los parámetros estructurales del capital como modo de control socio-metabólico" (p. 107), y tiene la función de rectificar, dentro de los límites del sistema del capital los defectos estructurales que se colocan como barreras para la acumulación.

Siendo así, para analizar los cambios en los mecanismos de protección social entender la dinámica capitalista es central. También las particularidades contemporáneas dela sociabilidad burguesa a partir del momento en que la crisis estructural explota serán determinaciones fundamentales para nuestras reflexiones ya que, en el contexto de crisis, el capital impone nuevas exigencias al Estado que, como comando político e inseparable de la base material del capital, debe asumir una participación creciente auxiliando a los grandes negocios. Hacen parte de las exigencias impuestas al capital la redefinición de las formas de organizar los mecanismos y estructuras destinadas a la producción y reproducción de la fuerza de trabajo.

Para alcanzar el objetivo de problematizar algunos de los elementos entendidos como centrales para analizar los cambios producidos en las políticas sociales en las últimas décadas, organizamos este artículo en dos partes. Iniciamos sistematizando las transformaciones societarias a partir de la crisis estructural del capital, buscando entender los cambios que se procesan en Estado y en las políticas sociales frente a las nuevas exigencias colocadas por el proceso de expansión del capital. Ese proceso contradictorio de transformaciones se encuentra permeado y tensionado por las luchas sociales que corporifican los diferentes intereses antagónicos inherentes al modo de producción capitalista. En una segunda parte, concentraremos la atención en las transformaciones que se procesan en la protección social y el destaque que asumen las acciones coercitivas, moralizadoras, represivas y controladoras del Estado, especialmente, en la amalgama de las acciones en el área de asistencia social y de seguridad.

\section{La sociabilidad burguesa y las determinaciones de la crisis estructural}

Para entender los cambios en las estructuras de protección social y las barreras que el orden del capital impone a los procesos de ampliación de las políticas sociales y de manutención de los derechos, comenzaremos por el conjunto de alteraciones que se encuentran en curso en las sociedades capitalistas, transformaciones que no pueden ser desvinculadas de las limitaciones que el capital encuentra para expandirse. Por eso, la crisis que inicia en los años 1960-70 será un marco fundamental para las reflexiones aquí desarrolladas.

Para Mészáros (2017) el sistema del capital es irreformable e incontrolable. Se trata de un sistema organizado vertical y jerárquicamente bajo el comando del capital; esa organización vertical será la garantía para perpetuar la subordinación estructural del trabajo al capital y proteger los intereses vitales del sistema facilitando la expansión apoyada en la explotación del trabajo. De esta forma, se trata de un sistema en el cual el poder controlado por el capital no puede ser compartido con fuerzas o grupos que pretenden trascenderlo. Se trata de un sistema incontrolable, cuya incontrolabilidad deriva de la propria naturaleza del capital y de su necesidad estructural y ontológica de expandirse a través de la extracción del valor. Como explicita Mészáros cuando analiza la experiencia soviética,

El capital es un modo de control que se sobrepone a todo lo demás, mismo antes de ser controlado - en un sentido apenas superficial - por los capitalistas privados (o más tarde, por funcionarios públicos del Estado de tipo soviético). Las peligrosas ilusiones de que se puede superar o 
Pastorini, A. Intersecciones entre las políticas sociales y las acciones de seguridad: elementos para analizar los cambios en la protección social en el contexto de crisis del capital.

subyugar el poder del capital por la expropiación legal/política de los capitalistas privados surgen cuando se deja de llevar en cuenta la real naturaleza del relacionamiento entre controlador y controlado. (Mészáros, 2002, p. 98)

El sistema del capital para expandirse precisa controlar toda la sociedad (instituciones políticas, el cuerpo jurídico, Estado, la producción, el consumo, las empresas, la economía etc.) y subordinarla a sus exigencias y necesidades. Así, la organización vertical y jerárquica será central para subordinar todas las funciones vitales a las exigencias de la expansión y de la acumulación.

El sistema sociometabólico del capital es constituido por una enorme red de contradicciones que lo acompañan desde siempre. ${ }^{4}$ Esas contradicciones o "defectos estructurales", en las palabras de Mészáros (2017), pueden ser administrados por algún tiempo, pero nunca superados de forma definitiva ya que remiten a la propia estructura del sistema del capital.

Como forma de administrar algunas de esas contradicciones en el interior de los limites últimos del capital, el sistema de reproducción social vigenteacciona diversas estrategias (por ejemplo, intensificación de la explotación de los trabajadores, nuevas formas de organizar y controlar el trabajo, ampliación del consumo, desarrollo de políticas sociales) que funcionan como válvulas de escape para contribuir con la expansión y acumulación. Esos recursos $y$ estrategias que buscan desplazar contradicciones solo podrán existir caso sean compatibles con el funcionamiento del sistema y con el desarrollo global, o sea, siempre que no atenten contra las bases y el funcionamiento del sociometabolismo

4 Hacen parte de esa red de contradicciones insuperables: producción/control, producción/ consumo, producción/circulación, competición/ monopolio, desarrollo/subdesarrollo, producción/ destrucción, expansión del empleo/generación de desempleo (Mészáros, 2017). actual (Mészáros, 2002).

Es importante destacar que este proceso contradictorio es atravesado y tensionado por las luchas sociales que dan materialidad a los diferentes intereses y antagonismos de clases y sectores de clases. La definición de estrategias y mecanismos de administración de las contradicciones y de los límites del sistema del capital, no es un proceso harmónico ni tranquilo.

En la constitución de la forma económica y de la forma política - y del entrelazamiento entre ambas - permea necesariamente la lucha de clases. [...] El establecimiento económico y político de las formas capitalistas es necesariamente conflictivo, contradictorio, no harmónico y repleto de crisis porque está fundado en explotaciones y dominios de clase y grupos. Es la lucha de clases se corporifica y constantemente tensiona y altera sus formas sociales correspondientes. Por lo tanto, solo es posible comprender la materialización de la forma política por medio de los variables y distintos movimientos de las luchas de clase. (Mascaro, 2016, p. 28)

Es así como variados elementos y factores inciden en la determinación de una configuración particular de los márgenes de las fronteras y posibilidades del capital. Entre esos elementos destacamos las luchas protagonizadas por los sectores subalternos y explotados, que en distintos momentos históricos asumen formas variadas (desde estrategias reformistas hasta revoluciones proletarias, pasando por inúmeras formas de resistencia y de oposición al capitalismo, al imperialismo y al capital) que en mayor o en menor medida buscan desafiar los límites del sistema vigente.

Es así como en función de la organización del capital y de las luchas sociales, la forma política estatal puede variar, y ha variado a lo largo del siglo $\mathrm{xx}$, asumiendo el formato de Estado 
democrático y, en otros momentos, una forma dictatorial o fascista.

El Estado con su forma política particular es una precondición para el funcionamiento constante del sistema del capital que requiere de una estructura de comando político con una función correctiva, que facilite la administración de las contradicciones y barreras que se interponen al proceso de acumulación. O sea, para Mészáros (2002) el Estado moderno es la "única estructura correctiva compatible con los parámetros estructurales del capital como modo de control sociometabólico" (p. 107).

De forma análoga, podríamos decir que las políticas sociales administradas por el Estado, que asumieron configuraciones y características variadas en los distintos momentos y contextos históricos, fueron (y continúan siendo) importantes mecanismos correctivos del sistema que permiten administrar y desplazar de forma temporaria algunas de las contradicciones que se colocan como barreras para el proceso de acumulación.

Entendidas así, estas políticas públicas también serán concebidas como históricamente determinadas y estructuralmente articuladas con las relaciones sociales dominantes; de esa forma, poseen un carácter contradictorio, histórico y transitorio.

Las políticas sociales cuandoatienden algunas de las necesidades concretas de los trabajadores, concretizan al mismo tiempo otras funciones que son fundamentales para la acumulación, tales como: administrar el desempleo y la pobreza, baratear la reproducción de la fuerza de trabajo, legitimar política e ideológicamente el orden social, socializar los costos, riesgos y las pérdidas de la producción etc. Las formas y características que las políticas sociales asumirán en los diferentes momentos históricos y en las diversas formaciones sociales se vinculan con las particularidades que asumen las contradicciones sociales. Estos mecanismos correctivos del Estado no son etenos y únicos, sino que los mismos pasan por importantes transformaciones que se expresan, por un lado, en sus características y formas de organización (principios orientadores, estructuración de las acciones, beneficios etc.) y, por otro lado, en las características de los sujetos destinatarios de estas.

Los sistemas de protección social que buscaban producir y reproducir la fuerza de trabajo, para ser incorporada al proceso de producción, a través de un conjunto de políticas sociales como salud, previsión, educación etc. y leyes laborales, por varias décadas contribuyeron para amortecer los impactos nocivos de las crisis cíclicas (caracterizadas generalmente como crisis de superproducción y superacumulación) ${ }^{5}$ administrando una parte de las contradicciones y límites del capital.

Sin embargo, cuando el período de prosperidad llega a su fin en el contexto de crisis estructural del capital a partir de los años 1960-70, esas estructuras de protección social comienzan a ser reformadas, una vez que el nuevo contexto de crisis afecta todas las instituciones políticas y exige alteraciones en las formas de intervención del Estado. Es a parir de ese momento que presenciamos importantes transformaciones en las políticas sociales.

Como explica Mészáros (2002) la crisis actual es una crisis estructural que posee un carácter universal, alcance global, una escala de tiempo extensa, continua y permanente y se expande de modo rastrero. Así es importante decir que,

(...) no es muy difícil imaginar las implicaciones de una crisis sistémica, verdaderamente estructural; (...) Bajo las condiciones de crisis estructural del capital, sus constituyentes destructivos avanzan con fuerza extrema, activando el espectro de la incontrolabilidad total en una forma que hace prever la autodestrucción,

5 Para profundizar el debate sobre la crisis del capital puede ser consultado: Mandel (1990) y Mészáros (2002). 
tanto para este sistema reproductivo social excepcional, en si, como para la humanidad en general. (Mészáros, 2002, p. 100)

Ese componente destructivo del sistema puede ser evidenciado, por ejemplo, en el aumento del consumo destructivo de la naturaleza - y de los recursos no renovables - y en el proceso de destructividad del trabajo (debido a la intensificación de la explotación, el deterioro de las condiciones de trabajo, aumento de relaciones de trabajo análogas a la esclavitud, las precarias condiciones de vida y de trabajo de las poblaciones inmigrantes, de los niños etc.).

O sea, las transformaciones por las que pasan las políticas sociales en las últimas décadas no pueden ser desvinculadas del proceso de crisis del capital y de las diferentes estrategias correctivas del Estado para crear las condiciones y facilidades para la expansión del capital.

\section{Los caminos de la reconfiguración de las políticas sociales}

Para avanzar en la discusión sobre las políticas de protección social partimos de la premisa que los cambios en la configuración de las políticas sociales, a partir de los años 1980-90, no pueden ser pensados separados de las transformaciones en la organización del capital y de las nuevas demandas que proceso de valorización le coloca al Estado. En las últimas décadas del siglo pasado comienza a percibirse el destaque y la centralidad que ocupan los programas de reducción de la pobreza (como, por ejemplo, los programas de transferencias monetarias).

A partir de los años 1990, con el avance de las reformas de orientación neoliberal, se busca poner la asistencia social, en el centro de la protección social, como la política estructuradora, perdiendo de esa forma su importancia como mecanismo de inclusión en el ámbito de la protección social, y abandonando su potencialidad en cuanto mecanismo articulador entre las diferentes políticas públicas. Al mismo tiempo, se pretende atribuirle la competencia de combatir la pobreza y las desigualdades sociales, capacidad que ni esta ni otra política social podrían asumir. (Patorini, 2016, p. 82)

Aquí nos guiaremos por la idea que estos programas focalizados en la pobreza y en la indigencia se constituyen en una pieza fundamental de la estrategia del Estado para administrar la superpoblación relativa. Tal como mencionamos anteriormente, en este contexto de crisis estructural se intensifican las contradicciones del sistema y se agravan los problemas ya existentes (como desempleo, pauperismo, subconsumo etc.) indicando el inicio de una nueva crisis del capital que presenta características distintas de las crisis cíclicas anteriores, colocándose como una verdadera crisis estructural y sistémica.

Ahora el capital para poder gestionar esa masa creciente de trabajadores desempleados, subempleados y ocupados de forma precarizada (situaciones que expresan el proceso de cronificación del desempleo) precisa organizar nuevas estrategias y revisitar e intensificar algunas de las ya existían, entre las que se destacan los programas de transferencia monetaria y las acciones en el área de la seguridad.

Mészáros explica que el desempleo

ya no se limita a un 'ejército de reserva' que se encuentra a la espera de ser activado e incorporado en el proceso de la expansión productiva del capital, algunas veces en una expansión prodigiosa. Ahora la grave realidad del desempleo deshumanizante asumió un carácter crónico, que hasta fue reconocido por los defensores más acríticos del capital como 'desempleo estructural', como forma de autojustificativa, como si éste 
nada tuviera que ver con la naturaleza perversa de su sistema adorado. (Mészáros, 2017, p. 22)

En el caso de América Latina donde el desempleo, la precarización y el subempleo siempre estuvieron presentes, la expansión de los programas de transferencia monetaria (generalmente condicionados) que se colocan el objetivo de controlar la pobreza es acompañada de la reducción del protagonismo del Estado como proveedor directo de otros servicios en el área de salud, educación y previsión. Esa reducción no implica en una retirada del Estado y si en la creación de mecanismo jurídicos y ventajas financieras que ayudan a estimular el proceso de ampliación de la presencia del sector privado en dichas áreas.

No podemos dejar de mencionar que esos cambios se articulan (en el tempo y en el espacio) con los procesos de reforma ${ }^{6}$ de los Estados y con la ampliación de los programas de privatización de las empresas y sectores públicos (Stein, 2008). Por un lado, los programas de privatización (directa e indirecta) producen significativas transformaciones en las políticas sociales (en especial, en elárea de la salud, educación y previsión) que pasan a convivir con el aumento de la participación del sector privado que ve al espacio de los servicios como importantes nichos de inversión y de valorización del capital. Por otro lado, las reformas (o las contrarreformas, según mencionamos antes) de los Estados crean nuevos mecanismos de gestión o amplían algunos de los ya existentes buscando facilitar el acceso al fondo público por parte del sector privado (Salvador, 2010). ${ }^{7}$

6 Aquí utilizamos el término contrarreforma para hacer referencia a las reformas neoliberales impuestas por los organismos multilaterales a los países localizados en la periferia del capitalismo, que implicaron en la reducción de los derechos sociales, laborales y, en algunos casos, también de los derechos políticos.

7 Eseconjunto de reformasy políticas macroeconómicas integran los denominados programas de "ajuste estructural" impuestos por los organismos multilaterales como FMI y el Banco Mundial a partir de la segunda mitad de los años 1980, en los denominados
Acompañando ese movimiento de reforma, también se observó, en las últimas décadas, una tendencia a aumentar las acciones y los programas coercitivos, represivos y punitivos que tienen como principales destinatarios a los sectores más empobrecidos. Y decimos aumentar estas acciones punitivas y coercitivas porque, como indica Cueva (1987), el autoritarismo es un trazo que siempre estuvo presente en las formaciones sociales latinoamericanas. Como menciona este autor "las estructuras capitalistas subdesarrolladas engendran un autoritarismo tendencial históricamente comprobable (digobien tendencialy no fatal)" (p. 61). Y haciendo referencia a los nexos entre las últimas dictaduras cívico-militares, que dominaron la región por décadas, y las bases estructurales de estas sociedades capitalistas, el mismo autor nos explica que

[seria] errado ver en aquellas dictaduras la simple presencia de una 'fuerza bruta', carente de un proyecto histórico de clase. [...] En este sentido, incluso el que los militares tratasen al pueblo como un ejército de ocupación trataría a un país ocupado, es un hecho execrable pero no necesariamente excepcional. De una parte, las oligarquías latinoamericanas siempre se consideraron verdaderas ocupantes de estos países de negros, indios, mestizos y mulatos (que no de ciudadanos); de otra parte, la modernización de nuestros ejércitos fue realizada exactamente en la escuela de los ocupantes de Argelia y Vietnam. No aprendieron, pues, a tratar a sus compatriotas como citoyens de París o como citizens newyorquinos, sino como a parias de la Casbah argelina o de las selvas de Indochina. (Cueva, 1987, p. 63-64)

países periféricos. En el área social, las reformas concentran las acciones en los programas de reducción de la pobreza absoluta y de la indigencia a través de los programas compensatorios, focalizados y paliativos. Esa estrategia se expande en la década de los 1990 y se consolida en la primera década del siglo XXI (Pastorini, 2016, p.74-75). 
Pastorini, A. Intersecciones entre las políticas sociales y las acciones de seguridad: elementos para analizar los cambios en la protección social en el contexto de crisis del capital.

Esos trazos discriminatorios, estigmatizantes y coercitivos impregnan fuertemente la estrategia punitiva que se constituye, por ejemplo, por los denominados programas de prevención social del delito, la retirada de población en situación de calle y de usuarios de alcohol y otras drogas, las estrategias de monitoreo, allanamientos y militarización del cotidiano de algunos barrios y espacios públicos etc. Estos programas y acciones muchas veces se encuentran vinculados a la denominada política de seguridad, pero otras veces integran las políticas de salud, desarrollo social, educación etc.

Más allá de la vinculación institucional de cada acción o programa, aquí queremos destacar que una parte significativa de éstos, a la hora de su ejecución, se combinan en los territorios donde viven los sectores más empobrecidos con las acciones y programas sociales destinados a los individuos y grupos en situación de vulnerabilidad. Así, ambos conjuntos de acciones (seguridad y asistencia para los grupos más empobrecidos) van constituyendo una importante estrategia del Estado para administrar la pobreza entendida como una amenaza al orden vigente, contribuyendo al mismo tiempo para reducir el carácter explosivo del desempleo crónico a través de la integración y del control de los sectores subalternos.

La utilización del criterio territorial/ geográfico pasa a ser central para consolidar una red de acciones y programas en el área de seguridad y de protección, que define como objetivo la manutención del orden público, la prevención de delitos, el combate al crimen organizado y la protección social, fiscalización y control de los grupos vulnerabilizados.

Las estrategias en el área de la seguridad pueden asumir formas variadas. En un extremo podemos identificar a las políticas públicas que concentran diversas actividades socioeducativas y preventivas de delitos con un fuerte carácter reintegrador, que buscan cambios en los comportamientos principalmente de los jóvenes que viven en los "barrios más pobres” o en las periferias urbanas. En el otro extremo podemos localizar diversas acciones represivas, violentas y militarizadas como encarcelamiento en masa, operativos policiales en las periferias, programas de monitoreo, ocupaciones militarizadas del poder público etc.

Es importante destacar que generalmente los destinatarios de las acciones en el área de seguridad y aquella concentradas en la denominada política de asistencia a los individuos vulnerabilizados son los mismos grupos sociales. Esa "coincidencia" se apoya los procesos de culpabilización de los sujetos por sus situaciones de necesidad y de criminalización de la pobreza.

A través de las identificaciones entre pobreza y violencia o entre pobreza y crimen, se va consolidando el proceso que estamos denominando aquí de criminalización de la pobreza. Este proceso se apoya en la identidad entre las situaciones de "vulneración" y riesgo social con las situaciones (y mismo, espacios y territorios) concebidos como violentos, criminosos y peligrosos. Así, los individuos y grupos que se son víctimas de las desigualdades sociales pasan a ser vistos como culpados y responsables de su situación de pobreza, desempleo, violación de derechos etc. (Patorini, 2018).

De esta forma queremos demostrar que esa combinación de acciones indica un cambio en la articulación de los aspectos coercitivo y consensual del Estado que se vale cada vez más del control y de la coerción para administrar la masa de trabajadores que excede las necesidades y demandas del proceso de producción. Estas alteraciones contribuyen con el rediseño de la protección social y condiciona la forma de implementar las políticas y programas públicos.

Estos procesos de cambio en las funciones correctivas del Estado no son mecánicos ni naturales, son parte central del proyecto defendido por los 
sectores dominantes que buscan facilitar la expansión del capital, creando nuevos espacios de valorización, reproduciendo una parte de los trabajadores para ser incorporados en el proceso de producción, controlando y monitoreando a la población excedente, reprimiendo y recluyendo en las cárceles a los sectores considerados "peligrosos".

Ese crecimiento de las estrategias punitivas y controladoras del Estado es justificado política e ideológicamente por los sectores conservadores a través del "discurso del miedo" frente al aumento de la violencia y del supuesto desorden público. Como bien explica Morás (2010), estas estrategias son facilitadas por la "extensión de las nuevas narrativas, actores y situaciones que promueven la inseguridad y elaboran una cultura del miedo como figura omnipresente (...). [Es así como] el miedo, la inseguridad y la violencia pasaron a ser importantes factores de cohesión social" (p. 16).

Sin embargo, no podemos desconsiderar que estos procesos no son naturales ni eternos y que se encuentran tensionados por las luchas sociales en las cuales las diferentes clases y sectores de clases buscan concretizar sus intereses antagónicos y proyectos en disputa.

Estos elementos nos indican que estamos ante un proceso de importantes transformaciones de las estrategias tradicionales utilizadas por el Estado para administrar las manifestaciones de la cuestión social que se traduce en la reducción de las acciones y programas de protección social y en la hipertrofia de los elementos coercitivos y represivo del poder público, reforzando el carácter punitivo del Estado.

\section{Conclusiones}

A partir de las reflexiones desarrolladas en este trabajo podemos concluir que los cambios que se encuentran em curso en las políticas sociales solamente podrán ser entendidos a partir del conjunto de transformaciones que tienen su raíz en la crisis estructural del capital que condicionarán la organización y la función reguladora y correctiva del Estado.

La ampliación del desempleo, el deterioro de las condiciones de producción y reproducción de la fuerza de trabajo, la precarización de las contrataciones y de las condiciones de vida de amplios sectores, la reducción y ataque a los derechos sociales, laborales y políticos son indicadores de las dificultades que el sistema del capital encuentra para expandirse. El aumento de la población sobrante será un elemento determinante para explicar las transformaciones en las políticas sociales que cambian sus características fundamentales, cuando el Estado concentra su actuación en lo social ampliando las acciones destinadas a atender las necesidades de los grupos más vulnerabilizados y reduciendo su papel de proveedor directoenáreas tradicionalmente concentradas en las manos del Estado (como la salud, educación y previsión). Ese proceso es complementado con la ampliación de las acciones coercitivas y punitivas administradas, mismo que no de forma exclusiva, por el poder público.

Esa realidad nos muestra como esa masa creciente de trabajadores superfluos y extremamente precarizados que sufre con la degradación de sus condiciones de vida, pasa a recibir un tratamiento cada vez más deshumanizante que se expresa en estrategias agresivas, como: legislaciones y normatizaciones autoritarias, uso de la fuerza y de la violencia, fuertes mecanismos de control, mayor rigidez de la penas y puniciones a los "pequeños delitos", ayudas sociales focalizadas en la pobreza con fuerte carga moralizadora, fiscalizadora y controladora.

\section{Referencias Bibliográficas}

Cueva, A. (1987). La cuestión democrática en América Latina: algunos temas y problemas. Revista Estudios Avanzados, 41-77.

Fleury, S. (1994). Estado sem cidadãos. Seguridade social na América Latina. Rio de Janeiro: Fiocruz. 
Mandel, E. (1990). A crise do capital. Os fatos e sua interpretação marxista. São Paulo: Unicap.

Mascaro, A. (2016). Estado e forma política. São Paulo: Boitempo Editorial.

Mészáros, I. (2002). Para além do capital. São Paulo: Boitempo Editorial.

Mészáros, I. (2017). O século XXI. Socialismo ou barbárie? São Paulo: Boitempo Editorial.

Morás, L. E. (2010) Los dilemas y desafíos de las políticas progresistas de seguridad. Revista Encuentros Uruguayos, (3), 6-21.

Pastorini, A. (2016). Consideraciones sobre las políticas sociales en América Latina: el proceso de asistencialización de la protección social. Revista Trabajo Social, (22-23), 167-190.

Pastorini, A. (2018). Criminalização e a administração da questão social no Brasil. In C. E. Montaño et al. (Ed.), Expressões da ofensiva ultraconservadora na conjuntura contemporânea (pp. 71-88). Rio de Janeiro: PPGSS/UFRJ.
Pastorini, A., \& Galizia, S. (2006). A redefinição do padrão de proteção social brasileiro. Revista Praia Vermelha, (14-15), 72-103.

Pastorini, A., \& Galizia, S. (2012). Principais características da redefinição da proteção social no Brasil. In A. Pastorini, A. A. Moraes, \& S. Galizia (Eds.), Estado e Cidadania. Reflexões sobre as políticas públicas no Brasil contemporâneo (pp. 103-136). Rio de Janeiro: FGV.

Salvador, E. (2010). Fundo públicoepolíticas sociais na crise do capitalismo. Serviço Social e Sociedade, (104), 605-631.

Stein, R. (2008). Os Programas de Transferência de Renda Latino Americanos: o que revelam e o que encobrem suas estratégias. Debates Sociais, (67-68), 131-146.

\section{Sobre la Autora}

Alejandra Pastorini

Profesora de la Escola de Serviço Social de la Universidade Federal do Rio de Janeiro (ESS-UFRJ).

Trabajadora Social (UdelaR-Uruguay), Magister y Doctorado en el Programa de Pós-Graduação em Serviço Social de la Universidade Federal do Rio de Janeiro (PPGSS-UFRJ) y Post-doctorado en la UFAL - Meceió. 